\title{
Digital metrics: A graph-theoretical approach
}

\author{
Frank HARARY \\ Department of Mathematics, University of Michigan, Ann Arbor, MI 48109, USA
}

\author{
Robert A. MELTER
}

Department of Mathematics, Southampton College of Long Island University, Southampton, NY11968, USA

\section{Ioan TOMESCU}

Faculty of Mathematics, University of Bucharest, Bucharest, Romania

Received 3 August 1983

\begin{abstract}
Consider the following two graphs $M$ and $N$, both with vertex set $Z \times Z$, where $Z$ is the set of all integers. In $M$, two vertices are adjacent when their euclidean distance is 1 , while in $N$, adjacency is obtained when the distance is either 1 or $\sqrt{2}$. By definition, $H$ is a metric subgraph of the graph $G$ if the distance between any two points of $H$ is the same as their distance in $G$. We determine all the metric subgraphs of $M$ and $N$. The graph-theoretical distances in $M$ and $N$ are equal respectively to the city block and chessboard matrics used in pattern recognition.
\end{abstract}

Key words: Digital metrics, graph theory, city block distance, chessboard distance.

\section{Introduction}

We follow the notation and terminology of the book [3]. A subgraph $H$ of $G$ is a metric subgraph if the distance between any two points of $H$ is the same as their distance in $G$. Graphs in which every connected induced subgraph is metric are said to be distance-hereditary. A characterization of distance-hereditary graphs was derived by Howorka [6]. (Two diagonals $e_{1}, e_{2}$ or a cycle $\varphi$ are called a pair of skew diagonals of $\varphi$ if the graph $\varphi+e_{1}+e_{2}$ is homeomorphic with $K_{4}$.) He showed, for example, that a graph $G$ is distance-hereditary if and only if each cycle of $G$ of length at least five has a pair of skew diagonals. (Figure 1 illustrates, as in [6], a distance-hereditary graph with 6 points.) Metric subgraphs have also been studied by Kundu [7] who showed that if $G$ has a unique metric spanning tree then $G$ is regular. He thus provided an answer to a question posed by Chartrand and Schuster [1]. Other results on isometric graphs are

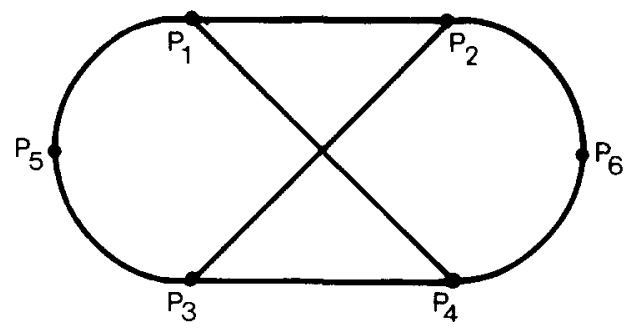

Fig. 1. A distance-hereditary graph.

due to Chartrand and Steward [2].

In work on pattern recognition (see [10]) one considers a variety of distances defined on $Z \times Z$, the set of all integral points in the plane. For example, the city block distance $d_{4}$ and chessboard distance $d_{8}$ are defined by

$$
\begin{aligned}
& d_{4}\left[\left(X_{1}, Y_{1}\right),\left(X_{2}, Y_{2}\right)\right]=\left|X_{1}-X_{2}\right|+\left|Y_{1}-Y_{2}\right|, \\
& d_{8}\left[\left(X_{1}, Y_{1}\right),\left(X_{2}, Y_{2}\right)\right]=\max \left(\left|X_{1}-X_{2}\right|,\left|Y_{1}-Y_{2}\right|\right) .
\end{aligned}
$$

Other distances for $Z \times Z$ have recently been studied in [8]. 
If $u, v$ are points of $Z \times Z$, then $d_{4}(u, v)$ and $d_{8}(u, v)$ are equal respectively to the usual graph theoretic distance in the graphs $M$ and $N$, both of which have $Z \times Z$ as vertex set. In $M$ two vertices are adjacent when their euclidean distance is 1 , while in $N$ adjacency is obtained when this distance is either 1 or $\sqrt{2}$. The graph $M$ is often called the Manhattan graph. One could refer to $N$ as a kind of diagonalized Manhattan graph. It can also be appropriately called the King's graph since adjacency is equivalent to two points being a King's move apart on an infinite chessboard. In Figure 2 we show some metric subgraphs of $M$ and $N$. Our object is to provide characterizations of the metric subgraphs of the Manhattan graph and the King's graph.

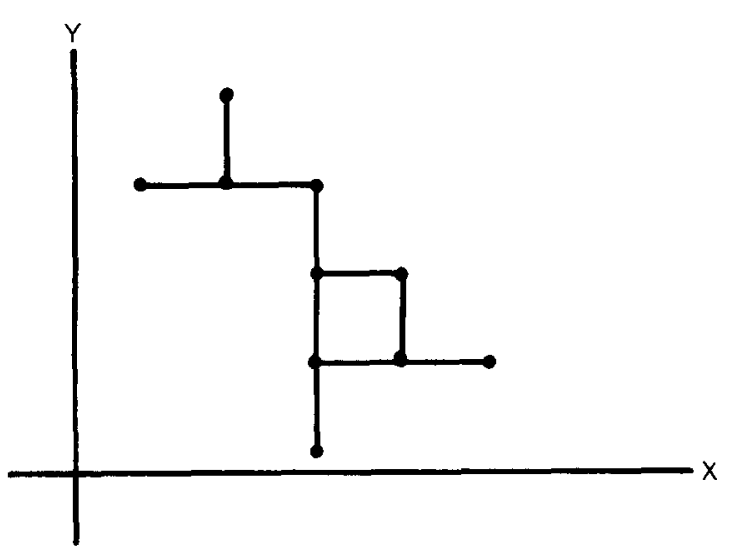

Fig. 2a. A metric subgraph of $M$ (this graph is axially convex but not diagonally convex).

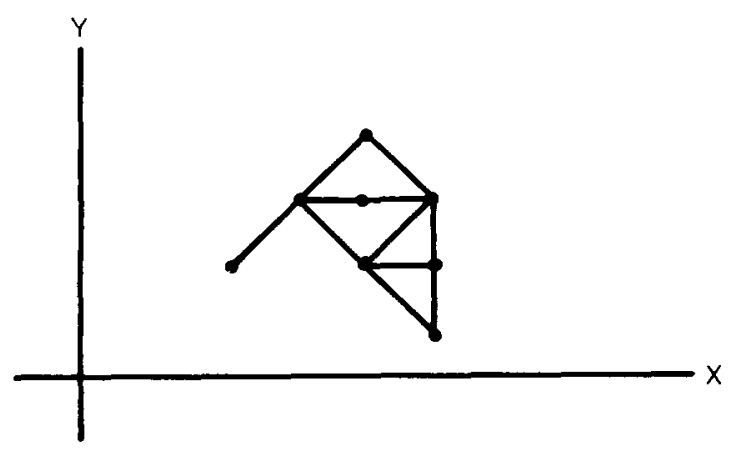

Fig. 2b. A metric subgraph of $N$ (this graph is diagonally convex but not axially convex).

\section{Metric subgraphs of the Manhattan graph}

A general notion of convexity in graphs has been defined by Harary and Nieminen [5]. A set $S \subset V(G)$ is convex if for all $u, v \in S$, every vertex on all $u-v$ geodesics is also in $S$. If $G$ were not mentioned in the preceding sentence, this definition would be the same as that of a convex set in any other metric space. It will be useful, however, to define the following related but different concept. A subgraph $G$ of $M$ is axially convex if for any two points of $G$ lying on a line parallel to the coordinate axes, all points on the line segment connecting them belong to $V(G)$.

Rosenfeld [9] characterized geodesics for $M$ in the following way: A path

$$
\left(X_{1}, Y_{1}\right),\left(X_{2}, Y_{2}\right), \ldots,\left(X_{n}, Y_{n}\right)
$$

of $M$ is a geodesic if and only if

$$
X_{1} \leq X_{2} \leq \cdots \leq X_{n} \text { and } Y_{1} \leq Y_{2} \leq \cdots \leq Y_{n} .
$$

We have assumed without loss of generality that $X_{1} \leq X_{n}$ and $Y_{1} \leq Y_{n}$.

We now proceed to the main theorem of this section.

Theorem 1. A subgraph $G$ of the Manhattan graph $M$ is a metric subgraph if and only if $G$ is both connected, and axially convex.

Proof. If $G$ is a metric subgraph of $M$, then $G$ obviously is connected. Suppose that $G$ is not axially convex. It follows that there are two points $a, b \in$ $V(G)$ such that the line through $a$ and $b$ is parallel to one of the coordinate axes, but at least one point of the segment connecting $a$ and $b$ does not belong to $V(G)$. This implies that $d_{G}(a, b) \geq d_{M}(a, b)+2$, which contradicts the hypothesis.

Suppose now that the subgraph $G$ is connected and axially convex. It remains to show that $d_{G}(a, b)=d_{M}(a, b)$ for any $a, b \in V(G)$. Since $G$ is connected there is a path in $G$ between any two points $a, b$ of $G$. Let a geodesic $P_{a b}$ be determined by the sequence of points

$$
a=\left(X_{1}, Y_{1}\right),\left(X_{2}, Y_{2}\right), \ldots,\left(X_{r}, Y_{r}\right)=b
$$


and suppose that $X_{1} \leq X_{r}, Y_{1} \leq Y_{r}$ and $d_{G}(a, b)>$ $d_{M}(a, b)$. Since $P_{a b}$ is not a geodesic for $M$ it follows that there is an index $s \geq 1$ such that

$$
X_{1} \leq X_{2} \leq \cdots \leq X_{s}, \quad Y_{1} \leq Y_{2} \leq \cdots \leq Y_{s}
$$

and

$$
X_{s}>X_{s+1} \text { or } Y_{s}>Y_{s+1} .
$$

We shall give details of the proof for the instance in which $X_{s}>S_{s+1}$. Since $P_{a b}$ is a geodesic in a subgraph of $M$, it follows that

$$
X_{s-1}=X_{s}=X_{s+1}+1 \text { and } Y_{s+1}=Y_{s}=Y_{s-1}+1 \text {. }
$$

We will examine separately the two cases I: $X_{1} \geq X_{s}$ and II: $X_{1}<X_{s}$.

Case I. If $X_{1}=X_{s}$ then, since $X_{1} \leq X_{r}$, there is a point $d=\left(X_{p}, Y_{p}\right)$ on $P_{a b}$ such that $p \leq r, X_{p}=X_{s}$, and $Y_{p}>Y_{s}$. If $c=\left(X_{s}, Y_{s}\right)$ and $G$ is axially convex it follows that all points on the segment connecting $c$ and $d$ are in $V(G)$ (see Figure 3). If the subpath

$$
\left(X_{s}, Y_{s}\right),\left(X_{s+1}, Y_{s+1}\right), \ldots,\left(X_{p}, Y_{p}\right)
$$

of $P_{a b}$ is replaced by the vertical path $P_{c d}$ between $c$ and $d$, then a path between $a$ and $b$ in $G$ is obtained which is shorter than $P_{a b}$; this contradicts the hypothesis that $P_{a b}$ is a geodesic in $G$.

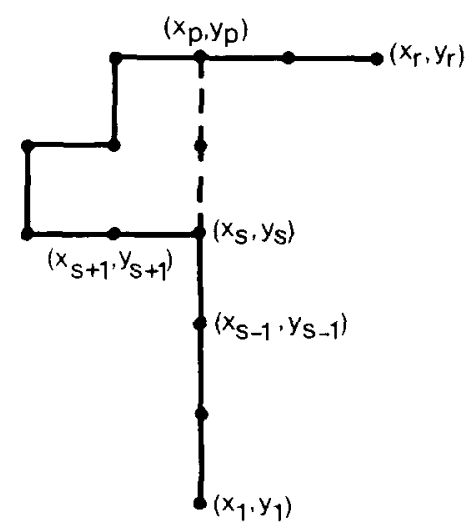

Fig. 3. Illustration for the proof of Theorem 1.

Case II. If $X_{1}<X_{s}$ then there is an index $1 \leq$ $p \leq s-2$ such that $X_{p}=X_{s+1}$ and $Y_{p}<Y_{s+1}$, i.e., the points $c=\left(X_{p}, Y_{p}\right)$ and $d=\left(X_{s+1}, Y_{s+1}\right)$ lie on a line parallel to the $y$-axis. We now replace the subpath

$$
\left(X_{p}, Y_{p}\right),\left(X_{p+1}, Y_{p+1}\right), \ldots,\left(X_{s+1}, Y_{s+1}\right)
$$

of $P_{a b}$ by the vertical path between $c$ and $d$; again, a path between $a$ and $b$ shorter than $P_{a b}$ has been constructed in $G$.

\section{Metric subgraphs of the King's graph}

The following variation of convexity is pertinent to the characterizations at hand. A subgraph $G$ of the King's graph $N$ is diagonally convex if for any two points of $G$ lying on a line with slope +1 , all points of the line segment connecting them belong to $V(G)$.

Rosenfeld [9] characterized geodesics in $N$ as follows: A path

$$
\left(X_{1}, Y_{1}\right),\left(X_{2}, Y_{2}\right), \ldots,\left(X_{n}, Y_{n}\right)
$$

of $N$ is a geodesic if and only if

$$
X_{1}<X_{2}<\cdots<X_{n} \text { or } Y_{1}<Y_{2}<\cdots<Y_{n},
$$

assuming without loss of generality that $X_{1} \leq X_{n}$ and $Y_{1} \leq Y_{n}$.

The principal result of this section can now be stated.

Theorem 2. A subgraph $G$ of the King's graph $N$ is a metric subgraph of $N$ if and only if $G$ is

(i) connected,

(ii) diagonally convex, and

(iii) $G$ does not contain as a subgraph any of the eight subgraphs illustrated in Figure 4.

Proof. It is clear that if $G$ does not satisfy any of (i), (ii), or (iii), then it is not a metric subgraph of $N$. In particular, in each of the graphs of Figure 4, we have

$$
d_{N}(C, D)=d_{G}(A, B)+1=d_{G}(C, D)-1 .
$$

Conversely assume that a subgraph $G$ of $N$ satisfies (i), (ii) and (iii) and is not a metric subgraph of $N$. It follows that there exist two points $u, v \in V(G)$ such that $d_{G}(u, v)>d_{N}(u, v)$. Since $G$ is connected, there is a shortest path $P_{u v}$ between $u$ and $v$. Let $P_{u v}$ be determined by the sequence of points

$$
u=\left(X_{1}, Y_{1}\right),\left(X_{2}, Y_{2}\right), \ldots,\left(X_{r}, Y_{r}\right)=v
$$

and suppose that $X_{1} \leq X_{r}$ and $Y_{1} \leq Y_{r}$. Since $P_{u v}$ is not a geodesic in $N$ it follows that there are indices $i, j, 1 \leq i, j \leq r-1$ such that 


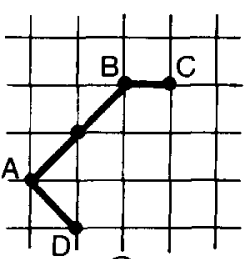

(a)

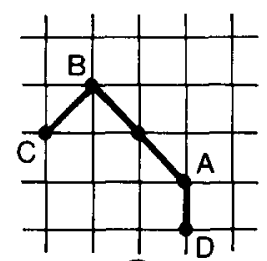

(e)

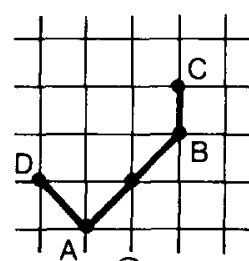

(b)

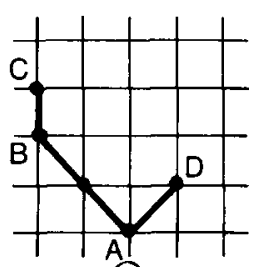

(f)

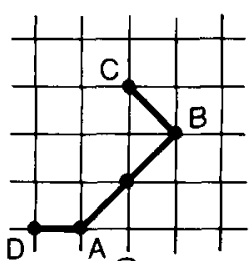

(C)

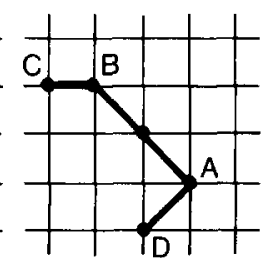

(g)

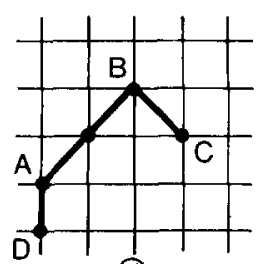

(d)

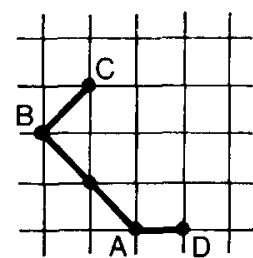

(b)

Fig. 4. Forbidden subgraphs in metric subgraphs of $N$.

$$
\begin{aligned}
& X_{1}<X_{2}<\cdots<X_{i}, \quad Y_{1}<Y_{2}<\cdots<Y_{j}, \\
& X_{i} \geq X_{i+1} \quad \text { and } \quad Y_{j} \geq Y_{j+1} .
\end{aligned}
$$

We need to consider separately the cases I: $i=j$ and II: $i \neq j$.

Case I. If $i=j$ then

$$
X_{i+1}=X_{i}-1 \text { and } Y_{i+1}=Y_{i}
$$

or

$$
X_{i+1}=X_{i} \quad \text { and } \quad Y_{i+1}=Y_{i}-1
$$

Since $X_{i-1}=X_{i}-1$ and $Y_{i-1}=Y_{i}-1$ it follows that in either case the path

$$
\left(X_{i-1}, Y_{i-1}\right),\left(X_{i+1}, Y_{i+1}\right)
$$

of length one in $G$ is shorter than the subpath

$$
\left(X_{i-1}, Y_{i-1}\right),\left(X_{i}, Y_{i}\right),\left(X_{i+1}, Y_{i+1}\right)
$$

of $P_{u v}$. This contradicts the assumption that $P_{u v}$ is a geodesic in $G$.

Case II. Assume without loss of generality that $i<j$. Since $X_{i+1} \leq X_{i}$ and $Y_{i+1}>Y_{i}$ it follows that

$$
X_{i+1}=X_{i} \text { and } Y_{i+1}=Y_{i}+1
$$

or

$$
X_{i+1}=X_{i}-1 \text { and } Y_{i+1}=Y_{i}+1
$$

There are in fact three subcases which must now be examined:
II.1. $X_{j}=X_{j-1}+1$ and $Y_{j}=Y_{j-1}+1$,
II.2. $X_{j}=X_{j-1}$ and $Y_{j}=Y_{j-1}+1$,
II.3. $X_{j}=X_{j-1}-1$ and $Y_{j}=Y_{j-1}+1$.

We shall present the details for subcase II.1. The other subcases can be dealt with in a similar manner.

In subcase II.1 since $Y_{j+1} \leq Y_{j}$ it follows that exactly one of the following statements is true:

( $\alpha) X_{j+1}=X_{j}-1$ and $Y_{j+1}=Y_{j}$,

(B) $X_{j+1}=X_{j}$ and $Y_{j+1}=Y_{j}-1$,

( $\gamma$ ) $X_{j+1}=X_{j}+1$ ziad $Y_{j+1}=Y_{j}$,

( $\delta) X_{j+1}=X_{j}+1$ and $Y_{j+1}=Y_{j}-1$.

If $(\alpha)$ or $(\beta)$ holds it is easy to see that, as in the proof of case I, we can replace a subpath of length two of $P_{u v}$ by a path of length one and hence $P_{u v}$ cannot be a geodesic for $G$.

If $(\gamma)$ is true then there exists an index $k$, $i+1 \leq k \leq j-1$ such that $X_{k}<X_{k+1}<\cdots<X_{j}, Y_{k}<$ $Y_{k+1}<\cdots<Y_{j}$, and either

( $\delta 1) X_{k-1}=X_{k}$ and $Y_{k-1}=Y_{k}-1$ or

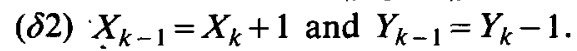

In the case of $(\delta 1)$, the slope of the line passing through the points

$$
C=\left(X_{j+1}, Y_{j+1}\right) \text { and } D=\left(X_{k-1}, Y_{k-1}\right)
$$

is equal to 1 . Since $G$ is diagonally convex it follows that

$$
d_{G}(C, D)=d_{P_{u v}}(C, D)-1
$$

and hence $P_{u v}$ cannot be a geodesic for $G$.

If $(\delta 2)$ holds then the subpath of $P_{u v}$ between $C$ and $D$ is similar to the graph depicted in Figure $4 \mathrm{a}$ and hence (iii) implies that

$$
\begin{aligned}
d_{G}(C, D) & =d_{G}(A, B)+1 \\
& <d_{G}(A, B)+2=d_{P_{u v}}(C, D)
\end{aligned}
$$


which contradicts the assumption that $P_{u v}$ is a geodesic.

When $\delta$ holds we can show the existence of an index $k$ having the same properties, but if

$$
X_{k-1}=X_{k} \text { and } Y_{k-1}=Y_{k}-1
$$

the graph of Figure $4 d$ is obtained. Finally if

$$
X_{k-1}=X_{k}+1 \text { and } Y_{k-1}=Y_{k}-1
$$

the slope of $C D$ equals 1 and it follows that $P_{u v}$ is not a geodesic for $G$ since $G$ is diagonally convex.

\section{References}

[1] Chartrand, G. and S. Schuster (1974). Which graphs have unique distance trees? Amer. Math. Monthly 81, 53-56.
[2] Chartrand, G. and M.J. Steward (1971). Geometric graphs. Springer Lecture Notes Math. No. 186, pp. 63-67.

[3] Harary, F. (1969). Graph Theory. Addison-Wesley, Reading, MA.

[4] Harary, F., Achievement and avoidance games. To appear.

[5] Harary, F. and J. Nieminen (1981). Convexity in graphs. J. Differential Geometry 16, 185-190.

[6] Howorka, E. (1977). A characterization of distance hereditary graphs. Quart. J. Math. Oxford 26, 417-420.

[7] Kundu, S. (1977). The Chartrand-Schuster conjecture: Graphs with unique distance trees are regular. J. Combin. Theory B 22, 233-245.

[8] Melter, R. and I. Tomescu (1983). Path-generated digital metrics. Pattern Recognition Letters 1, 151-154.

[9] Rosenfeld, A. (1978). Geodesics in digital pictures. Information and Control 36, 74-84.

[10] Rosenfeld, A. and A.C. Kak (1976). Digital Picture Processing. Academic Press, New York. 